\title{
LE CHLORDIAZEPOXYDE COMME AGENT PREANESTHESIQUE
}

\author{
Toussatnt Bouchard, M.D., Fernand Tanguay, M.D., et Yves Brgault, M.D.
}

\section{INTRODUCTION}

QUELS QUE SOIENT les bienfaits apportés à un patient par la médecine moderne, l'occurrence d'une condition chirurgicale, où le patient se voit aux prises à la fois avec le chirurgien et l'anesthésiste, garde certainement quelque chose d'inquietant pour ce patient. Il est donc juste de considérer que toute procédure qui, $1^{\circ}$ diminuera l'anxiété du patient, $2^{\circ}$ diminuera au minimum les risques inhérents à l'anesthésie, $3^{\circ}$ facilitera le travail du chirurgien, est une arme importante qui ne demande qu'à être perfectionnée.

Plusieurs catégories de médications préanesthésiques ont été employées au cours des années. Les combinaisons morphine-atropine, morphine-scopolamine, et surtout mépéridine-atropine, ont fait leurs preuves.

Depuis quelques années, les tranquillisants ont envahi la thérapeutique et toutes les spécialités ont eu à étudier leur action et leur influence dans leur sphère respective. Il va sans dire que l'anesthésiologie a cherché à bénéficier de leur application, spécialement si l'on tient compte du fait que l'un des buts primordiaux de la médication préanesthésique est de calmer l’anxiété du patient.

Le but du présent communiqué, est de faire un rapport préliminaire sur l'emploi du chlordiazépoxyde comme médication préanesthésique.

Les rapports concernant le chlordiazépoxyde ont commencé à atteindre le monde médical vers 1960 . Une importante littérature est maintenant accumulée au sujet de ce médicament en psychiatrie, en alcoolisme et en médecine interne. Quant à l'usage du composé comme prémédication, Brandt ${ }^{1}$ et Tornetta ${ }^{2}$ en ont publié d'excellentes études contrôlées utilisant la méthode à double insu.

\section{MatírRtal et MÉthode}

Les avancés du présent rapport, sont la suite de l'expérience cumulée par les membres du Service d'anesthésie de l'Hôpital Général St-Vincent-de-Paul, entre les mois de août 1963 et avril 1965. Durant cette période la prémédication chlordiazépoxyde-atropine a été utilisée chez environ 70 pour cent des adultes et la préparation usuelle mépéridine-atropine dans 30 pour cent. Aucune contreindication n'a été mise en cause. Parmi les 9,000 anesthésies de cas de chirurgie abdominale, osseuse, gynécologique, oto-rhino-laryngologique etc. environ 6500 ont reçu du chlordiazépoxyde.

A partir de ce vaste matériel clinique, des tendances ont été observées empiriquement sur les effets de ce médicament et confirmées relativement en choisissant

"Nom générique du "Librium," Hoffmann-La Roche Limitée.

†Service d'anesthésie, Hôpital Général St.-Vincent-de-Paul, Sherbrooke. 
BOUCHARD et al:: LE CHLORODIAZÉPOXYDE COMME AGENT PRÉANESTHÉSIQUE

100 patients au hasard parmi les feuilles d'observation complétées. Ces 100 patients demeurent constants pour tous les tableaux utilisés pour illustrer les différents stages pré- per- et post-anesthésiques.

\section{Dosage et ADMmistration}

Le dosage d'une façon générale était le suivant:

1. Chez les adultes. (a) Au coucher, $50 \mathrm{mg}$. per os.; (b) 45 minutes avant l'opération, chlordiazépoxyde $100 \mathrm{mg}$., atropine $1 / 150 \mathrm{gm}$. i.m.

2. Chez les personnes ágées 65 ans et plus. (a) Au coucher, 25 mg. per os.; (b) 45 minutes avant l'opération, chlordiazépoxyde 75 a $100 \mathrm{mg}$,, atropine $1 / 300 \mathrm{gm}$. i.m.

Dans certains cas d'obésité, $150 \mathrm{mg}$. de chlordiazépoxyde ont été donnés préopératoirement, mais l'effet a été sensiblement le même que chez les obèses qui n’avaient reçu que la dose usuelle (Fig. 1).

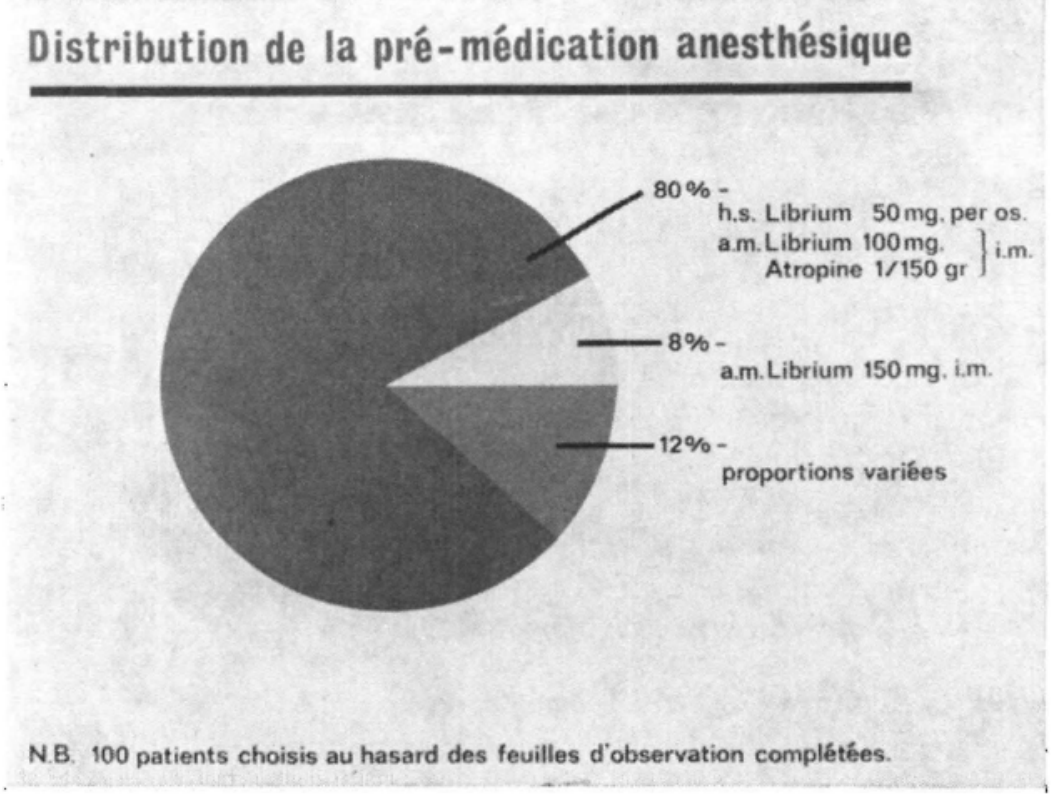

FIGURE 1

Voici donc d'après le consensus général des membres du service, nos opinions concernant ce médicament et portant sur: (1) la nuit pré-opératoire, (2) létat pré-opératoire immédiat, (3) les effets per-opératoires, (4) la période postopératoire.

\section{Nuit Pré-opératoire (Fig. 2)}

Sauf dans de très rares exceptions, tous les patients ayant reçu du chlordiazépoxyde au coucher, aux doses précitées, nous ont confié une fois à la salle d'opération, qu'ils avaient passé une nuit calme, sans rêves ou cauchemars et 


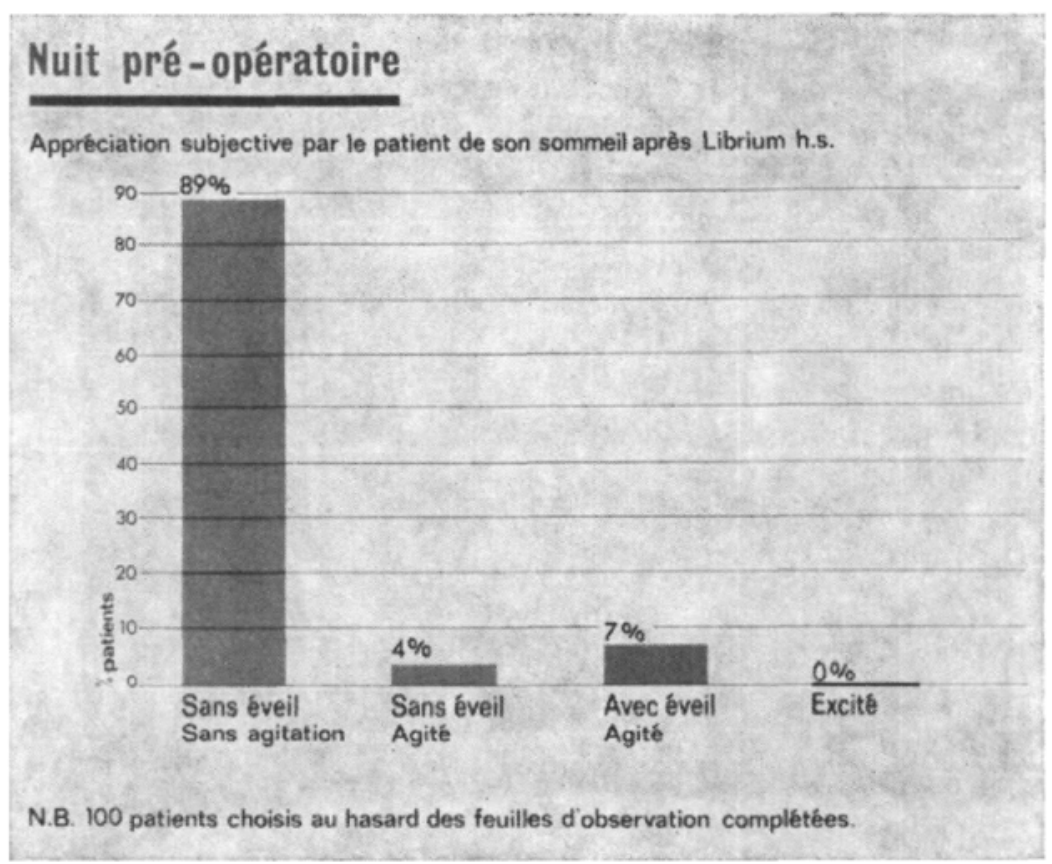

Figure 2

s'étaient éveillés frais et dispos. Les quelques exceptions à cette règle s'étaient éveillés assez tôt le matin mais ne se sentaient pas incommodés par cette insomnie et n'avaient ressenti aucune anxiété durant les quelques heures qui avaient précédé leur injection du matin.

A une ou deux reprises, des personnes âgées ont manifesté une somnolence prolongée qui a rendu inutile l'emploi de la dose pré-opératoire.

\section{L'Etat Pré-operatoire Immédiat (Fig. 3)}

1. Ce qui frappe d'abord chez le patient prêt à être anesthésié, c'est l'absence complète d'anxiété associée à une absence remarquable de somnolence. A première vue, cet état éveillé et alerte du patient nous laisse croire que la pré-médication n'a pas agi, mais si l'on questionne le patient, on se rend compte et il nous affirme, qu'il est bien détendu.

2. Même si le patient exprime une certaine crainte, elle est plutôt de type rationnel qu'émotif, en ce sens qu'aucune altération du pouls, de la pression artérielle, ou de la respiration ne se manifeste.

3. Il découle de tout ceci, que la coopération du malade est excellente pour prendre les différentes postures qu'on lui demande.

4. Il semble même qu'on puisse attribuer des vertus analgésiques au chlordiazépoxyde. En effet les patients tolèrent très bien les injections généralement douloureuses, au dos de la main ou au poignet, sans rétraction des membres ou autre geste réflexe. 


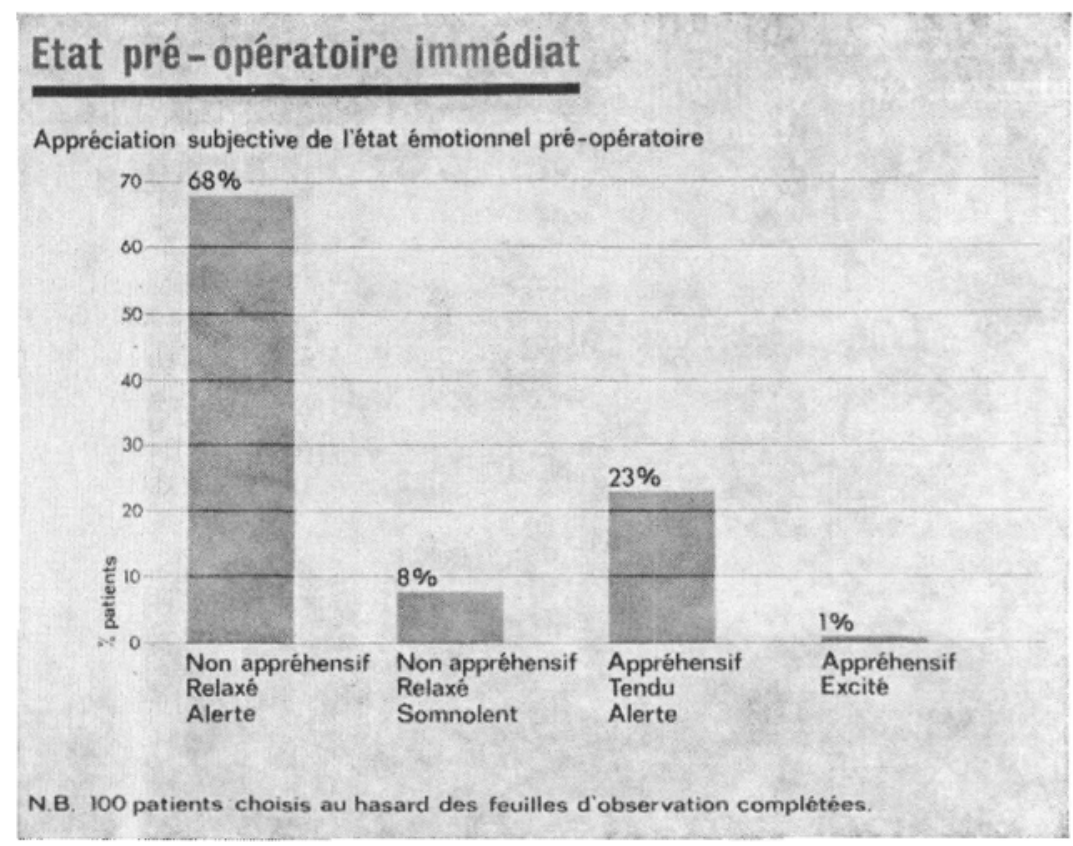

FIGURE 3

5. Par exception, aux doses employées, quelques cas (peut-être 1\%) se sont comportés tout à fait comme s'ils n'avaient reçu aucune médication. Dans tous ces cas, il s'agissait de patients souffrant déjà d'anxiété ou d'excitation névrotique et même psychotique.

\section{Effets Per-opératoires (Fig. 4)}

1. En cours danesthésie locale ou régionale (bloc, rachi, péridurale), nous avons surtout remarqué: (a) Le grand calme des patients qui tolèrent très bien des interventions même prolongées. (b) L'absence de dépression respiratoire qui a rendu inutile l'emploi d'oxygène en masque Elliot. (c) Une plus grande stabilité de la pression artérielle. (d) Un meilleur relâchement des muscles paravertébraux, rendant ainsi plus facile linjection épidurale ou spinale.

2. En anesthésie générale, les méthodes employées dans le service sont les suivantes: (a) Induction au thiopentone (ou au masque pour les personnes âgées). (b) Maintien au halothane, méthoxyflurane et surtout mélange azéotropique halothane-éther.

Dans ces cas, indépendemment de l'agent employé, nous avons retenu les impressions suivantes:

a. La dose de thiopentone requise pour obtenir une bonne induction, n'a pas varié comparativement d la dose requise chez les patients ayant reçu de la mépéridine.

b. Il semble que la baisse de la pression artérielle au moment de l'induction fut moins marquée qu'avec la mépéridine. En moyenne cette baisse ne dépasse pas 10 à $15 \mathrm{~mm}$. de mercure et elle est passagère. 


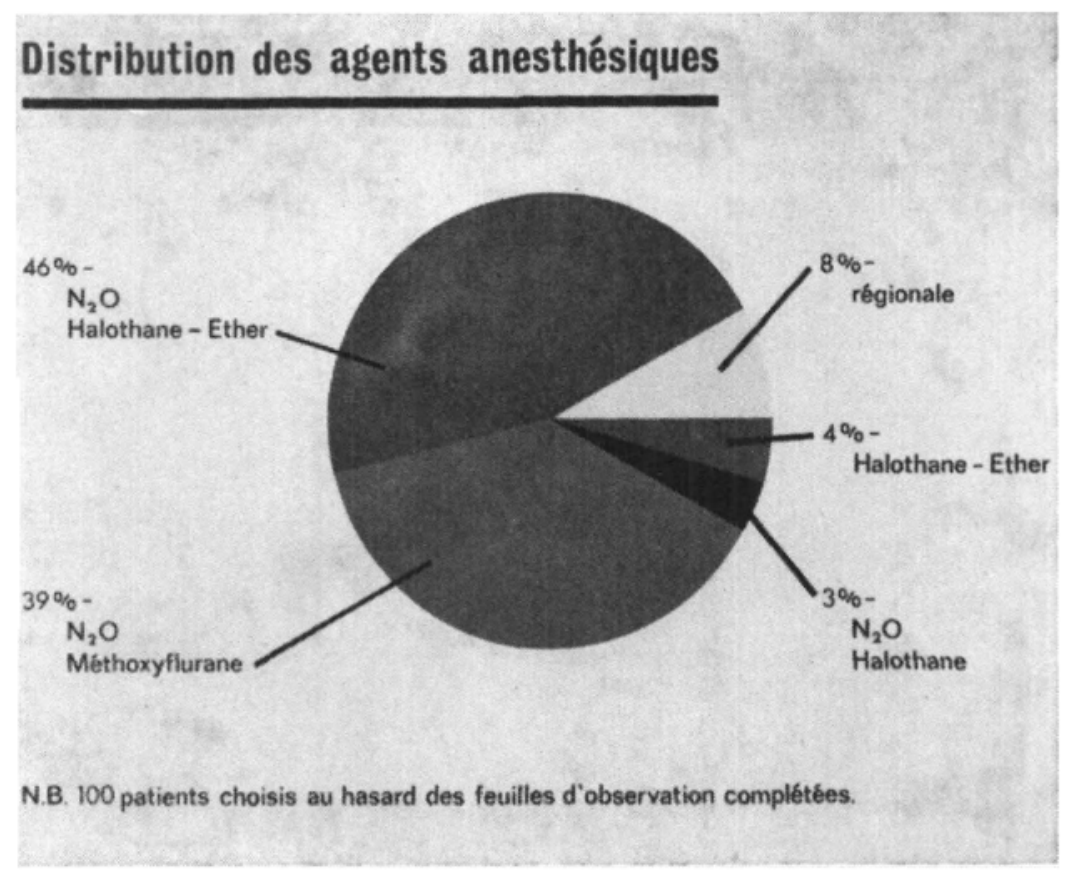

Figure 4

Chez les hypertendus d'origine émotive, la chute est naturellement plus marquée, mais le retour à la pression normale (c.a.d. le facteur émotif en moins) se fait tout aussi rapidement. La stabilité de la pression artérielle est excellente quel que soit l'agent anesthésique employé ou l'intervention pratiquée.

c. Le chlordiazépoxyde ne déprime pas la respiration chez le patient éveillé et ne semble pas non plus la déprimer chez le patient anesthésié. La fréquence et l'amplitude des mouvements respiratoires sont meilleures qu'avec la mépéridine.

$d$. Chose très importante pour le chirurgien, le relâchement musculaire du patient est définitivement favorisé par le chlordiazépoxyde, de sorte que, aux concentrations usuelles d'anesthésiques, des doses réduites de curarisants sont suffisantes même pour les interventions les plus difficiles.

\section{Effets Post-opératoires (Fig. 5)}

1. Le réveil lui-même est très calme et assez rapide, compte tenu de l'agent anesthésique employé. Les chutes de tension artérielle sont moins marquées.

2. Fait important à noter, les doses de calmants employés à la salle de réveil ont semblé diminuer de façon appréciable depuis l'emploi du chlordiazépoxyde pré-opératoire, diminuant d'autant le danger d'une dépression respiratoire qui avait été évitée en cours d'anesthésie.

3. Dans quelques rares cas, chez des patients très anxieux, une agitation marquée a accompagné le réveil. De nouvelles doses de chlordiazépoxyde n'ont pas calmé le malade, mais ont même semblé l'agiter davantage. II semble bien que nous ayons été ici en face d'une réaction paradoxale, comparable à celle rencontrée avec la mépéridine ou le phénobarbital. 


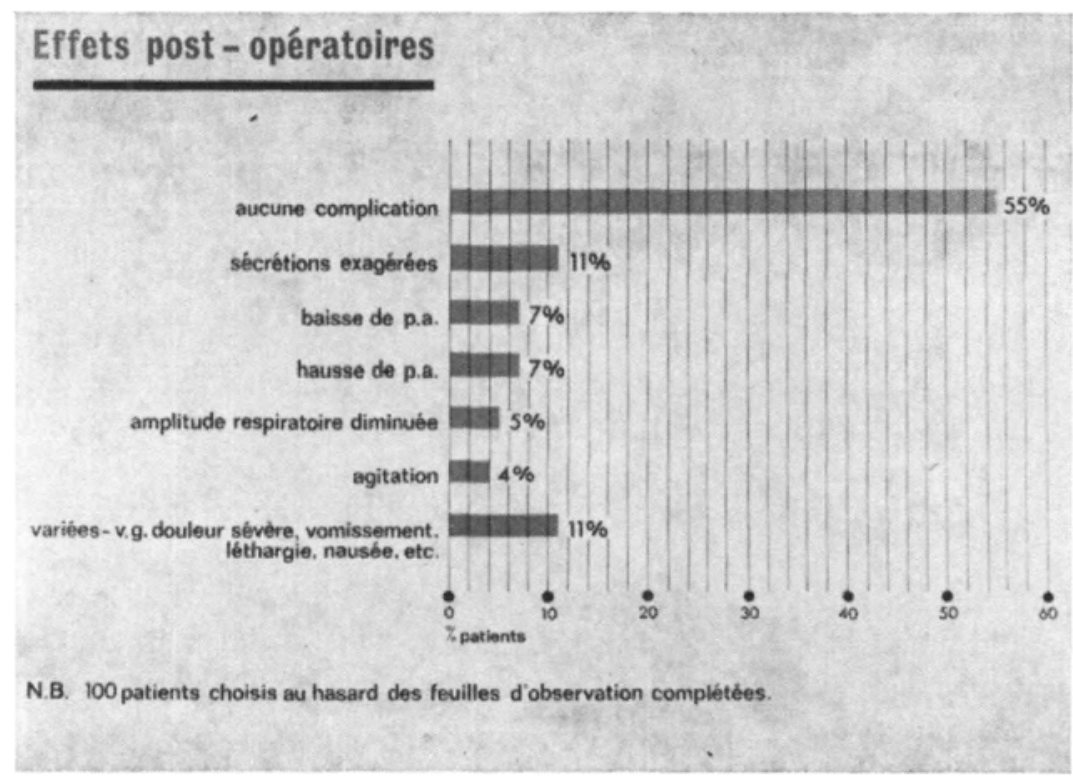

Figure 5

\section{Conclusion}

Le chlordiazépoxyde s'est avéré une aide précieuse comme agent préanesthésique en association avec l'atropine. Nous croyons que, comparé à la mépéridineatropine, les avantages sont les suivants: (1) excellente inhibition de l'anxiété; (2) absence de dépression circulatoire; (3) absence de dépression respiratoire; (4) action adjuvante à la myorésolution; (5) potentialisation (clinique) des analgésiques.

\section{REMERCIEMENT}

Le chlordiazépoxyde "Librium Roche" a été fourni gracieusement par la compagnie Hoffmann-La Roche Limitée.

\section{BIBLIOGRAPHIE}

1. Brandt, A. L. \& Lur, S. Y. Trial of Chlordiazepoxide as Preanesthetic Medication. Anesth. \& Analg. 41: 557. (1962).

2. Tornetra, F. J. Clinical Evaluation of Injectable Librium in Preanesthetic Medication. Anesth. \& Analg. 42: 4, 463 (1963). 